\title{
Smooth chaotic maps with zero topological entropy
}

\author{
M. MISIUREWICZ AND J. SMÍTAL
}

Institute of Mathematics, Warsaw University, PKiN IX p., 00-901 Warsaw, Poland, Institute of Mathematics, Komensky University, 84215 Bratislava, Czechoslovakia

\section{(Received 13 March 1987)}

Abstract. We find a class of $C^{\infty}$ maps of an interval with zero topological entropy and chaotic in the sense of $\mathrm{Li}$ and Yorke.

\section{Introduction}

Throughout this paper $f: I \rightarrow I$ will be a continuous map of a compact real interval $I$ into itself.

The notion of chaos has been introduced by $\mathrm{Li}$ and Yorke [5]. The following equivalent definition is given in [9] (cf. also [3]):

$f$ is chaotic if there is some $\varepsilon>0$ and a non-empty perfect set $S \subset I$ such that for any $x, y \in S, x \neq y$, and any periodic point $p$ of $f$,

$$
\begin{aligned}
& \limsup _{n \rightarrow \infty}\left|f^{n}(x)-f^{n}(y)\right| \geq \varepsilon, \\
& \liminf _{n \rightarrow \infty}\left|f^{n}(x)-f^{n}(y)\right|=0, \\
& \limsup _{n \rightarrow \infty}\left|f^{n}(x)-f^{n}(p)\right| \geq \varepsilon,
\end{aligned}
$$

where $f^{n}$ denotes the $n$th iterate of $f$. The set $S$ is called an $\varepsilon$-scrambled set for $f$.

It turns out that the chaos in the sense of $\mathrm{Li}$ and Yorke is a weaker property than positive topological entropy (and is equivalent to the property that the map has a trajectory which is not approximable by cycles, cf. $[3,9])$. An example showing this is given in [9].

The main aim of the present paper is to give simple examples of chaotic maps with zero entropy.

\section{Preliminary constructions}

We recall that the $\omega$-limit set of $x \in I$, denoted by $\omega_{f}(x)$, is the set of limit points of the sequence $\left(f^{n}(x)\right)_{n=0}^{\infty}$.

The following theorem will be useful in the sequel:

THEOREM 1. If there is a point $x \in I$ such that the set $\omega_{f}(x)$ is infinite and $f$ is not injective on $\omega_{f}(x)$ then $f$ is chaotic in the sense of Li and Yorke.

Proof. Choose $a, b \in \omega_{f}(x)$ such that $a \neq b, f(a)=f(b)$. Then any periodic neighbourhoods $J_{a}, J_{b}$ of $a, b$, respectively, must have a point in common. By [3], this implies that $f$ is chaotic. 
Remark 1. The condition of Theorem 1 is not necessary, an example is given in [9].

Recall that a continuous map $f: I \rightarrow I$, where $I=[a, b]$, is called unimodal (cf. [2]) if there exists $c \in(a, b)$ such that $f$ is strictly increasing on $[a, c]$ and strictly decreasing on $[c, b]$. We shall call $f$ weakly unimodal if there exists $c \in(a, b)$ such that $f$ is non-decreasing on $[a, c]$ and non-increasing on $[c, b]$.

Let $f$ be weakly unimodal. We shall say that $x, y \in I$ are equivalent (denoted by $x \sim y$ ) if there exists $n \geq 1$ such that $f^{n}$ is constant on $[x, y]$. Clearly, $\sim$ is an equivalence relation. Let $\tilde{I}=I / \sim$ be the factor space obtained by identifying to a point each equivalence class. These classes are closed intervals (possibly degenerated to a point) and thus $\tilde{I}$ is a closed interval (also possibly degenerated). The natural projection $\pi: I \rightarrow \tilde{I}$ is continuous and non-decreasing. Since $f$ is continuous, $x \sim y$ implies $f(x) \sim f(y)$. Therefore there exists a unique map $\tilde{f}: \tilde{I} \rightarrow \tilde{I}$ such that

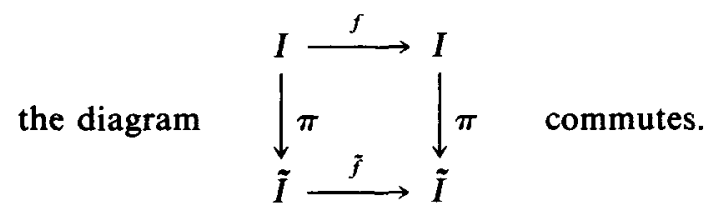

This $\tilde{f}$ is continuous and either monotone or unimodal.

By the period of a periodic point (orbit) we shall understand its smallest period.

LeMma 1. (a) If $x \in I$ is a periodic point of $f$ of period $k$ then $\pi(x)$ is a periodic point of $\tilde{f}$ of period $k$.

(b) If $y \in \tilde{I}$ is a periodic point of $\tilde{f}$ of period $k$ then there exists a unique periodic point $x \in I$ of $f$ for which $\pi(x)=y$. The period of $x$ is $k$.

Proof. (b) Let $\pi^{-1}(y)=[a, b]$. Then (4) and the equality $\tilde{f}^{k}(y)=y$ imply that $f^{k}([a, b]) \subset[a, b]$. Since $f$ is continuous, there is some $x \in[a, b]$ with $f^{k}(x)=x$. Clearly $\pi(x)=y$. The point $x$ cannot have a period $i<k$ because then by (4), $y$ would have period $i$.

Let $z \in[a, b]$. Since $z \sim x$, there is some $r$ such that $f^{r k}(z)=f^{r k}(x)=x$. This shows that $x$ is the unique periodic point of $f$ in $[\mathrm{a}, \mathrm{b}]$.

(a) From (4) it follows that $\tilde{f}^{k}(\pi(x))=\pi(x)$. The period of $\pi(x)$ cannot be $i<k$ because then, by (b), the period of $x$ would be also $i$.

Let $\mathscr{F}$ be the class of all weakly unimodal maps $f$, for which

the set $J_{f}=\{x \in I ; f(x) \geq f(y)$ for all $y \in I\}$ consists

of more than one point,

for each $n \geq 0, \quad f$ has a periodic orbit of period $2^{n}$,

$f$ has no periodic orbits of other periods.

LEMMA 2. If $f \in \mathscr{F}$ then $\tilde{f}$ is unimodal and satisfies (6) and (7).

Proof. By Lemma 1, the sets of periods of periodic orbits of $f$ and $\tilde{f}$ are equal. Therefore, if $f \in \mathscr{F}$ then $\tilde{f}$ satisfies (6) and (7). Then, since $\tilde{f}$ has periodic points of period larger than 2 , it cannot be monotone. Consequently, it is unimodal. 


\section{Main results}

First we prove the following

THEOREM 2. Any mapping $f \in \mathscr{F}$ has topological entropy zero and is chaotic in the sense of Li and Yorke.

Proof. Let $f \in \mathscr{F}$. By (7) and [8], $f$ has topological entropy zero.

By Lemma 2, $\tilde{f}$ is unimodal and satisfies (6) and (7). Therefore it has the same kneading invariant as the Feigenbaum map $\Phi$ (see e.g. [4 Proposition 4.6] for the uniqueness of this kneading invariant, and [2] for the description of the Feigenbaum map). Hence the relative positions of the turning point, its images and the periodic points are the same for $\Phi$ and $\tilde{f}$. Since $\pi$ is non-decreasing, by Lemma 1 it is the same also for $f$. However, for $\Phi$ this relative position is well-known. Let $c$ be the critical point of $\Phi$ and let $a_{n}$ be the periodic point of $\Phi$ of period $2^{n}$ with the largest image under $\Phi$. Then from the known properties of $\Phi$ we immediately get

$$
\begin{aligned}
\Phi^{2^{1}}(c) & <a_{1}<\Phi^{2^{3}}(c)<a_{3}<\cdots<c<\cdots<a_{4}<\Phi^{2^{4}}(c) \\
& <a_{2}<\Phi^{2^{2}}(c)<a_{0}<\Phi^{2^{0}}(c) .
\end{aligned}
$$

Therefore if $b_{n}$ is the periodic point of $f$ of period $2^{n}$ with the largest image under $f$ and $d \in J_{f}$ (see (5)) then

$$
\begin{aligned}
f^{2^{1}}(d) & <b_{1}<f^{2^{3}}(d)<b_{3}<\cdots<d<\cdots<b_{4}<f^{2^{4}}(d) \\
& <b_{2}<f^{2^{2}}(d)<b_{0}<f^{2^{0}}(d) .
\end{aligned}
$$

Let $d_{1}=\lim _{n \rightarrow \infty} b_{2 n+1}, d_{2}=\lim _{n \rightarrow \infty} b_{2 n}$. Since

$$
\Phi\left(a_{0}\right)<\Phi\left(a_{1}\right)<\Phi\left(a_{2}\right)<\Phi\left(a_{3}\right)<\cdots<\Phi(c),
$$

we have also

$$
f\left(b_{0}\right)<f\left(b_{1}\right)<f\left(b_{2}\right)<f\left(b_{3}\right)<\cdots<f(d)
$$

and therefore

$$
\lim _{n \rightarrow \infty} f\left(b_{i}\right)=f\left(d_{1}\right)=f\left(d_{2}\right) \leq f(d)
$$

Since

$$
\lim _{n \rightarrow \infty} \Phi\left(a_{n}\right)=\lim _{n \rightarrow \infty} \Phi^{2^{n+1}}(c)=\Phi(c)
$$

and $c$ is not periodic for $\Phi$, the $\tilde{f}$-itineraries of all points

$$
y \in\left[\lim _{n \rightarrow \infty} \tilde{f}\left(\pi\left(b_{n}\right)\right), \tilde{f}(\pi(d))\right]
$$

are the same (in fact, from the result of [6] applied to a slightly modified map $f$ it follows that the above interval is degenerated to a point; however, we do not need to use it). By (9), this means that we can replace in ( 8$) d$ by $d_{i}, i=1,2$. Consequently

$$
d_{i} \in \omega_{f}\left(f\left(d_{1}\right)\right) \quad \text { for } i=1,2 .
$$

By the definition of $d_{1}$ and by (8), $d_{1}$ is not periodic. Hence $\omega_{f}\left(f\left(d_{1}\right)\right)$, which by (10) contains the whole trajectory of $d_{1}$, is infinite. The point $d$ in (8) is an arbitrary element of $J_{f}$ and hence $J_{f} \subset\left[d_{1}, d_{2}\right]$. Therefore by (5), $d_{1}<d_{2}$. By (9) $f\left(d_{1}\right)=f\left(d_{2}\right)$, and hence by Theorem $1, f$ is chaotic. 
THEOREM 3. F contains a $C^{\infty}$ map.

Proof. There exists a $C^{\infty}$ map $g$ of $[0,1]$ onto $[0,1]$ which is weakly unimodal, satisfies (5) and $g(0)=g(1)=0$. Set $g_{\lambda}(x)=\lambda g(x)$ for all $\lambda, x \in[0,1]$. The map $g_{\lambda}$ is of class $C^{\infty}$ for each $\lambda$. By [7], the set $A=\left\{\lambda ; g_{\lambda}\right.$ satisfies (6) $\}$ is closed. Clearly, $g_{1}$ satisfies (6) but $g_{0}$ does not. Therefore if $\mu=\inf A$ then $\mu>0$ and $g_{\mu}$ satisfies (6).

Suppose that $g_{\mu}$ does not satisfy (7). Then by [1], if $\lambda$ is sufficiently close to $\mu$ then $g_{\lambda}$ satisfies (6), a contradiction. Clearly, $g_{\mu}$ satisfies also (5).

Remark 3. Let $\varepsilon$ be the length of $J_{g}$. Then $g_{\mu}$ has a non-empty $\varepsilon$-scrambled set $S$ (cf. [9]). Clearly $\varepsilon$ can be made arbitrarily close to 1 , but less than 1 . This result cannot be improved, since if $f:[0,1] \rightarrow[0,1]$ is a continuous map with zero topological entropy satisfying (1) and (2) for some $x, y \in[0,1]$ then $\varepsilon<1$. To see it note that in this case at least one of the sets $\omega_{f}(x), \omega_{f}(y)$, say $\omega_{f}(x)$, must be infinite. Let $I_{0}, I_{1}$ be disjoint closed periodic intervals covering $\omega_{f}(x)$ and such that $I_{0} \cap$ $\omega_{f}(x) \neq \varnothing \neq I_{1} \cap \omega_{f}(x)$ (cf. e.g. [9]). By (2) there exists some $z \in \omega_{f}(x) \cap \omega_{f}(y)$ and this $z$ cannot be periodic (cf. [9]). Consequently, $\omega_{f}(y) \subset I_{0} \cup I_{1}$ and since dist $\left\{I_{0}, I_{1}\right\}>0$, (2) implies that for some $m, f^{m}(x), f^{m}(y) \in I_{0}$. Hence for every $n \geq m, f^{n}(x), f^{n}(y)$ belong to the same interval $I_{i(n)}$, where $i(n) \in\{0,1\}$. But then

$$
\limsup _{n \rightarrow \infty}\left|f^{n}(x)-f^{n}(y)\right| \leq \max \left\{\operatorname{diam} I_{0}, \operatorname{diam} I_{1}\right\}<1 \text {. }
$$

Remark 4. Let $h$ be the tent map $(h(x)=1-|2 x-1|)$ and let $h_{\lambda}(x)=\min (\lambda, h(x))$ for $\lambda, x \in[0,1]$. For each $n \geqq 0$ let $\lambda_{n} \in[0,1]$ be the minimal number with the property that $\left[0, \lambda_{n}\right]$ contains a periodic orbit of $h$ of period $2^{n}$. Clearly $\lambda_{0}<\lambda_{1}<\cdots$. Put $\nu=\lim _{n \rightarrow \infty} \lambda_{n}(=0.8249080 \ldots)$. Then $h_{\nu} \in \mathscr{F}$. In such a way we obtain another simple example of chaotic map with zero topological entropy.

\section{REFERENCES}

[1] L. Block. Stability of periodic orbits in the theorem of Šarkovskii. Proc. Amer. Math. Soc. 81 (1981), 555-562.

[2] P. Collet \& J.-P. Eckmann. Iterated Maps on the Interval as Dynamical Systems. Birkhauser: Boston, 1980.

[3] K. Janková \& J. Smítal. A characterization of chaos. Bull. Austral. Math. Soc. 34 (1986), 283-292.

[4] L. Jonker. Periodic orbits and kneading invariants. Proc. London Math. Soc. 39 (3) (1979), 428-450.

[5] T. Y. Li \& J. A. Yorke. Period three implies chaos. Amer. Math. Monthly 82 (1975), 985-992.

[6] W. de Melo \& S. van Strien. A structure theorem in one dimensional dynamics. Informes de Matématica Série A-063/86, Rio de Janeiro (preprint 1986).

[7] M. Misiurewicz. Structure of mappings of an interval with zero entropy. Publ. Math. IHES 53 (1981), 5-16.

[8] M. Misiurewicz \& W. Szlenk. Entropy of piecewise monotone maps. Studia Math. 67 (1980), 45-63.

[9] J. Smital. Chaotic functions with zero topological entropy. Trans. Amer. Math. Soc. 297 (1986), 269-282. 